\section{Storage: Jurisdictional conflicts and state options - ADDENDUM}

doi: https://doi.org/10.1557/mre.2019.9

The following footnote should be included in this article [1]:

This paper was commissioned and accepted for publication by Elizabeth Kocs, who served as Editor-in-Chief of this journal from 2015-2018.
Nancy Lange, and Ted Thomas

REFERENCE:

1. Lange N., \& Thomas T. (2019). Storage: Jurisdictional conflicts and state options. MRS Energy \& Sustainability, 6, E4. Cambridge University Press. 